Research article

\title{
2, 4-Diamino-6- hydroxy pyrimidine inhibits NSAIDs induced nitrosyl-complex EPR signals and ulcer in rat jejunum S Somasundaram*1, R Simpson ${ }^{2}$, S Rafi ${ }^{2}$, JK Shergill ${ }^{3}$, I Bjarnason ${ }^{2}$ and J Wrigglesworth ${ }^{3}$
}

Address: ${ }^{1}$ Lineberger Comprehensive Cancer Center, CB\#7295, University of North Carolina, Chapel Hill. N.C 27599, ${ }^{2}$ Departments of Digestive Diseases, Kings College Hospital School of Medicine, London SE5 9PJ, UK and ${ }^{3}$ Centre for the Study of Metals In Biology and Medicine, King's College, London, SE1 8WA, UK

E-mail: S Somasundaram* - sivasoma@med.unc.edu; R Simpson - robert.simpson@kcl.ac.uk; S Rafi - sue.rafi@kcl.ac.uk; JK Shergill - j.shergill@kcl.ac.uk; I Bjarnason - ingvar.bjarnason@kcl.ac.uk; J Wrigglesworth - john.wrigglesworth@kcl.ac.uk ${ }^{*}$ Corresponding author

Published: 18 April 2002

BMC Gastroenterology 2002, 2:8
Received: 30 December 200I

Accepted: 18 April 2002

This article is available from: http://www.biomedcentral.com/I47I-230X/2/8

(C) 2002 Somasundaram et al; licensee BioMed Central Ltd. Verbatim copying and redistribution of this article are permitted in any medium for any purpose, provided this notice is preserved along with the article's original URL.

\begin{abstract}
Background: It has been suggested that one aspect of non-steroidal anti-inflammatory drugs induced intestinal damage is due to either uncoupling of mitochondrial oxidative phosphorylation or inhibition of electron transport. We investigated the latter possibility using electron paramagnetic resonance spectroscopy.

Results: Electron paramagnetic studies of NSAIDS on sub-mitochondrial particles revealed that indomethacin, but not with nabumetone, bound to a site near to Complex I and ubiquinone to generate a radical species. Normal rats exhibited prominent [3Fe-4S]ox signals $(g \sim 2.0 \mathrm{I})$ at $20 \mathrm{~K}$. One hour after indomethacin there was a prominent, intense and broad absorption pattern at ( $\mathrm{g}$ 2.07) suggesting, appearance of radical species overlapping [3Fe-4S]ox and was unaffected by pretreatment with 2,4 diamino -6-hydroxy pyrimidine. At $24 \mathrm{hrs}$, when macroscopic ulcers were seen, there was a new signal due to a nitric oxide radical (NO•). In contrast, nabumetone and 2,4 diamino-6-hydroxy pyrimidine pre-treated animals receiving indomethacin exhibited electron paramagnetic resonance spectra identical to those of controls at $24 \mathrm{hrs}$ and neither was associated with small intestinal ulcers. Indomethacin and 2,4 diamino hydroxy pyrimidine pre-treated rats, but not nabumetone, had increased intestinal permeability.
\end{abstract}

Conclusion: The results suggest that the in vivo effects of indomethacin modulate the mitochondrial respiratory chain directly at I h and $24 \mathrm{~h}$ through formation of nitric oxide. NO• appears to play an important role in the late pathogenic stages of NSAID enteropathy and may be the site for targeted treatment to reduce their toxicity.

\section{Introduction}

The major problem with the use of nonsteroidal anti-inflammatory drugs (NSAIDs) is the high prevalence of their gastrointestinal toxicity [1]. Accordingly $40 \%$ of patients have non-ulcer gastric lesions, $10-25 \%$ gastric ulcer and $65 \%$ NSAID enteropathy [2]. The development of safer NSAIDs is dependent on knowledge of the pathogenesis of damage. In general, two distinct phases (topical and 
systemic) are suggested to be involved in the pathogenesis of enteropathy [3-5]. The topical phase of damage may be due to the effect of NSAIDs to uncouple mitochondrial oxidative phosphorylation or inhibit electron transfer [46] either of which would result in a decrease in cellular ATP levels[7] and a disrupted intestinal barrier function. Consequently, it is suggested, increased intestinal permeability transforms the cellular damage into a tissue reaction where inflammation and ulcers occur because of mucosal exposure of luminal aggressive factors (such as acid, pepsin, bile, pancreatic secretions and bacteria) and the effects of decreased mucosal prostaglandins on the microvasculature.

In respect of early pathogenic events in the development of NSAID damage it has been shown that uncoupling of oxidative phosphorylation and inhibition of electron transport occurs with all commonly available acidicNSAIDs. Our earlier studies indicate $[8,9]$ that indomethacin, aspirin and naproxen uncouple mitochondrial oxidative phosphorylation at concentration between 30 and $500 \mu \mathrm{M}$ and inhibit the electron transport chain at higher concentrations. The issue of inhibition of electron transport by NSAIDs in the pathogenesis of damage and its pathophysiological consequences is complicated by the fact that nitric oxide $(\mathrm{NO} \bullet$ ), which plays a pathogenic role in the damage, may bind to metalloproteins [Fe-S] of the respiratory chain and by itself increase intestinal permeability [10]. Thus NSAIDs may inhibit electron transport directly or indirectly by induction of nitric oxide $(\mathrm{NO} \bullet)$ [11-16]. The aim of the current study was to assess and discriminate between the direct and indirect effects of NSAIDs on electron transport of the rat small intestine using electron paramagnetic resonance (EPR) spectroscopy.

Principle:Intestinal mitochondrial electron transfer system plays a significant role in the pathogenesis of NSAID enteropathy. In the mitochondria electron transfer occur in the iron sulphur proteins arranged sequentially. Loss (oxidation) and gain of electrons (reduction) in this iron sulphur proteins can be visualized as a spectrum in the EPR machine. It has been hypothesized that NSAID might have an affinity towards these iron sulphur proteins and alter the redox state.

\section{Materials and methods \\ Experimental design \\ Electron paramagnetic studies}

Male Sprague-Dawley rats (200-250 g) were used throughout EPR studies. 12 rats received indomethacin 20 $\mathrm{mg} / \mathrm{kg}$, which is a commonly used dose to induce consistent small intestinal damage in the rat (characterized by mitochondrial damage on electron microscopy within an hour,[17] neutrophil infiltration of mucosa on light microscopy at 3-5 hours and macroscopic lesions (ulcers) at
12-18 hours [18]); . A separate group of 12 rats received the non-acidic pro-NSAID nabumetone $(500 \mathrm{mg} / \mathrm{kg})$. The maximum human doses of indomethacin in man is 150 $\mathrm{mg} /$ day and $2000 \mathrm{mg}$ for nabumetone so that the equivalent doses given here are at least 8 and 17 fold greater than the recommended doses for indomethacin and nabumetone, respectively and many times higher than this required for anti-inflammatory activity.

Nabumetone is unusual amongst NSAIDs in that it does not produce any small intestinal lesions in the rat, presumably because it is non-acidic (and hence not a proton translocator or an inhibitor of the electron transport chain) and its active component 6-methoxy-2-naphthylacetic acid (6-MNA) is not subjected to excretion in bile $[9,19]$. Incorporation of nabumetone therefore allows assessment of the systemic effects of cyclooxygenase inhibition in isolation from its topical effects.

In separate studies the inhibitor of inducible nitric oxide synthase, 2,4 diamino-6-hydroxy pyrimidine (DAHP; a potent inhibitor of tetrahydrobiopterin synthesis, which is a cofactor for the inducible nitric oxide synthase activity) $1.0 \mathrm{gm} / \mathrm{kg}$ b.w. was administered to 12 rats intra-peritonealy $1 \mathrm{hr}$ before indomethacin $(20 \mathrm{mg} / \mathrm{kg})$ gavages.

Preparation of drugs: Indomethacin and nabumetone were dissolved in $10 \%$ dimethyl sulfoxide and then diluted to the final concentration of $5 \%$ and administered orally by a gastric gavage without anaesthesia. Control rats were received the same volume and concentrations of dimethylsulfoxide.

\section{Intestinal permeability assessment}

Rats, 12 in each group, were fasted overnight and received solvent, $20 \mathrm{mg} / \mathrm{kg}$ indomethacin or nabumetone $(500$ $\mathrm{mg} / \mathrm{kg})$ by gavage. 12 rats received DAHP i.p. $(1.0 \mathrm{~g} / \mathrm{kg}) 1$ $\mathrm{hr}$ before indomethacin $(20 \mathrm{mg} / \mathrm{kg})$. Rats were fed $2 \mathrm{hrs}$ later and fasted the following night. Twenty hrs after NSAID administration these rats received the intestinal permeability marker 51Cr- EDTA $(10 \mathrm{uCi} /$ rat in $0.5 \mathrm{ml}$ followed, by $0.5 \mathrm{ml}$ water) by gavage and were placed in metabolic cages. Urine was collected for 5 hrs and the samples were counted in Wallace 1842-LKB gamma counter with appropriate standard. The percentage of the orally administered radioactive chromium excreted in urine was calculated.

\section{Morphological assessment}

20 hours after administration of these drugs rat small intestinal mucosa $(n=12)$ was exposed by a cut through the anti- mesenteric side and laid out each group on a piece of cork for assessment of macroscopic damage.[24] The assessments were carried out by an independent person unaware of the drug treatments. An ulcer count was made to 
distinguish between pointed $(<5 \mathrm{~mm})$ and longitudinal (>5 mm) ulcers.

\section{Isolation of mitochondrial fractions}

Bovine heart mitochondria were isolated based on a modification of Schneider \& Hogeboom method (1950) [20], diluted to $10 \mathrm{mg}$ protein/ $\mathrm{ml}$ and sonicated in $3 \mathrm{ml}$ aliquots on ice with eight $15 \mathrm{sec}$ bursts, interrupted by $15 \mathrm{sec}$ intervals, at 18 amps using a micro tip probe. The sonicated suspension was centrifuged at $26,000 \mathrm{~g}$ for $10 \mathrm{~min}$ at 4 'C. The supernatant fraction was centrifuged at 130,000 $\mathrm{g}$ for $1 \mathrm{hr}$, and the sub mitochondrial particles pellet resuspended for EPR analysis after checking the purity of this fraction (by studying the electron transfer reactions using potassium ferricyanide as an electron acceptor and succinate as a substrate). This experiment was repeated for three times at various intervals. It has been attempted to isolate submitochondrial fraction of jejunal mitochondria from rats. In most of the cases we could not able to achieve a good yield of submitochodrial particles. Hence we used bovine heart miotochondria to understand the basic phenomena of NSAID binding sites.

\section{EPR spectroscopy}

$1 \mathrm{hr}$ and $24 \mathrm{hr}$ after administration of the drugs, the animals were anaesthetized (Hypnovil and Hypnorm) and underwent laparotomy. Segments of jejunum $10 \mathrm{~cm}$ distal to the ligament of Trietz were snap frozen with liquid nitrogen and stored for a maximum of 2 weeks. Samples were thawed and homogenized in $10 \%(\mathrm{w} / \mathrm{v})$ of sucrose (74 mM), mannitol (225 mM), homogenising buffer $(0.25 \mathrm{M})$ containing EDTA $(1 \mathrm{mM}), 5 \mathrm{mg} / \mathrm{ml}$ BSA solution in MOPS-NaOH buffer ( $10 \mathrm{mM}), \mathrm{pH} 7.4$, by 15 strokes of a tight fitting teflon pestle. $100 \mathrm{ul}$ samples ( $1 \mathrm{mg}$ protein) were placed in quartz EPR tubes (3 $\mathrm{mm}$-internal diameter) and immediately frozen in liquid nitrogen. X-band EPR spectra were recorded on a Bruker ESP 300 spectrometer fitted with an Oxford Instruments ESR900 liquid he- lium flow cryostat at $8 \mathrm{~K}$ and $20 \mathrm{~K}$ or $30 \mathrm{~K}$. The effect of 10 $\mathrm{ul}$ of $250 \mathrm{mM}$ indomethacin added to $90 \mathrm{ul}(1 \mathrm{mg}$ protein) of submitochondrial particles in a quartz EPR tube and read the EPR spectra at $8 \mathrm{~K}$ was assessed with appropriate controls. Spectra were an average of six scans and normalized for direct comparison. The experiments were repeated for three different times with separate group of animals.

EPR conditions: Temperature $8 \mathrm{~K}$ or $20 \mathrm{~K}$ or $30 \mathrm{~K}$ : microwave power $20 \mathrm{~mW}$, microwave frequency $9.35 \mathrm{GHz}$, modulation frequency $100 \mathrm{Khs}$, modulation amplitude 1 $\mathrm{mT}$, receiver gain $1.25 \times 10^{5}$, time constant $0.33 \mathrm{sec}$, sweep time $2.4 \mathrm{mT} / \mathrm{sec}$. Spectra displayed are average of 6-8 scans.

\section{Statistics}

Data are presented as mean \pm SEM. Statistical comparison between groups were made by Mann-Whitney U test.

\section{Results \\ Morphology}

No macroscopic changes were observed in controls or with indomethacin and nabumetone $1 \mathrm{hr}$ after drug administration. At $24 \mathrm{hr}$ the indomethacin-treated group exhibited severe intestinal ulceration (pointed ulcers: mean $=55 \pm 10$, longitudinal ulcers: mean $=15 \pm 9$ ). No ulcers were seen in controls, nabumetone or DAHP treated indomethacin gavaged rats. [Table 1]

\section{Intestinal permeability}

Controls excreted $2.3 \pm 1.2 \%$ of the orally administered 51Cr-EDTA in $5 \mathrm{~h}$ urine. Intestinal permeability was not significantly $(\mathrm{p}>0.5)$ increased following nabumetone (mean $2.7 \pm 1.5 \%$ ). Indomethacin treated rats and those pre-treated with DAHP (+indomethacin) had significantly increased $(\mathrm{p}<0.01)$ intestinal permeability to 51Cr-EDTA $(10.5 \pm 2.5 \%$ and $11.5 \pm 1.8 \%$ respectively) [Table 1 ]

Table I: The ulcerogenic and intestinal permeability (5 I Cr-EDTA excretion) effect of DMSO (Control), indomethacin, DAHP pretreated indomethacin and nabumetone gavaged rat small intestine.

\begin{tabular}{|c|c|c|c|c|}
\hline Group & Treatment & Pointed ulcers & Longitudinal ulcers & $\begin{array}{l}\% \text { Excretion of }{ }^{51} \mathrm{Cr} \text {-EDTA in } \\
\text { Urine }\end{array}$ \\
\hline I & Control(DMSO) & 0 & 0 & $2.3 \pm 1.2$ \\
\hline 2 & Indomethacin & $55 \pm 10$ & $15 \pm 9$ & ${ }^{*} 10.5 \pm 2.5$ \\
\hline 3 & DAHP pretreated indomethacin & 0 & 0 & $* 11.5 \pm 0.8$ \\
\hline 4 & Nabumetone & 0 & 0 & $2.7 \pm 1.5$ \\
\hline
\end{tabular}

\footnotetext{
$* P<0.01$
} 


\section{EPR spectroscopic study of NSAIDs on sub-mitochondrial particles}

At $8 \mathrm{~K}$, the EPR spectrum of bovine heart sub mitochondrial particles administered DMSO alone (Fig. 1) displayed a prominent resonance at $\mathrm{g} \sim 2.014$. This signal was only observed in the oxidized state at temperatures below $25 \mathrm{~K}$, and is characteristic of the [3Fe-4S] ox cluster of mitochondrial Complex II Centre S3. Extra features at $\mathrm{g}$ $\sim 2.04,1.99$ are present, and are assigned to interactions between ubisemiquinone radicals [23].

Indomethacin but not nabumetone or DAHP added to the sub mitchondrial particles significantly decreased the intensity of the latter featrures (Fig. 1) ( $\mathrm{g}$ 2.04 and 1.99) suggesting formation of radical species which is indicative of binding of indomethacin to and the inhibition of the respiratory chain direct to this site.

\section{EPR spectroscopic studies of NSAIDs on rat jejunal ho- mogenates}

At $20 \mathrm{~K}$, control rat intestinal homogenates also displayed a signal indicative of Centre S3, albeit weaker (Fig. 2a). A similar signal could be detected $1 \mathrm{hr}$ and $24 \mathrm{hr}$ after nabumetone administration. [Fig. 2b and Fig 3b)] However, with indomethacin the [3Fe-4S] ox signal intensity was decreased at $1 \mathrm{hr}$, and by $24 \mathrm{hr}$ the EPR spectrum of intestinal homogenates displayed a prominent new resonance centered at $\mathrm{g} \sim 2.0$, with a peak at $\mathrm{g} \sim 2.07$ and a triplet splitting centered at $\mathrm{g} \sim 2.01$, indicative of nitrosylhaem (haem-NO) formation. [Fig. 2c and Fig 3c]. DAHP pre treated indomethacin group significantly decreased the haem-NO intensity at $24 \mathrm{hr}$ after administration [Fig. $4]$, thereby allowing detection of the Centre S3 signal at 8 $\mathrm{K}$ [Fig. 5]. DAHP pretreated indomethacin group displayed the similar signals at $1 \mathrm{hr}$, when compared to indomethacin alone treated group [.i.e., decreased in the [3Fe-4S] ox signal at $1 \mathrm{hr}$, Data not shown]. The haem-NO signal observed in Fig. 3c shows a splitting of A $(14 \mathrm{~N}) \sim$ $330 \mathrm{mT}$ [Fig. 4 at $30 \mathrm{~K}$ ]. Similar signals have been observed for the NO-adducts of type II haem proteins such as cytochrome c oxidase and hemoglobin [21] Figure 3 explains the unique features of nitrosyl signals $(g \sim 2.04)$ at $20 \mathrm{~K}$ only in indomethacin treated group and not in the DMSO-control or nabumetone group. Figure 4 displays the differential pattern of the nitrosyl signals (i)-(ii) at $30 \mathrm{~K}$ after $24 \mathrm{hr}$ of indomethacin (i) and DAHP treated indomethacin group(ii) when compare to the DMSO-control (iii) spectra. This prominent nitrosyl signals at $30 \mathrm{~K}$ is the characteristics of the free radical species and temperature sensitive when compare to the signals at $8 \mathrm{~K}$, displayed in Figure 5. In indomethacin alone treated group (Figure 4) this broad signal displayed a peak at $\mathrm{g} \sim 2.04$, a distinct trough at $\mathrm{g} \sim 1.98$ and complex splitting (triplet) centered at $\mathrm{g} \sim 2.01$ at temperature above $25 \mathrm{~K}$, indicative of haem-nitrosyl formation.[21]

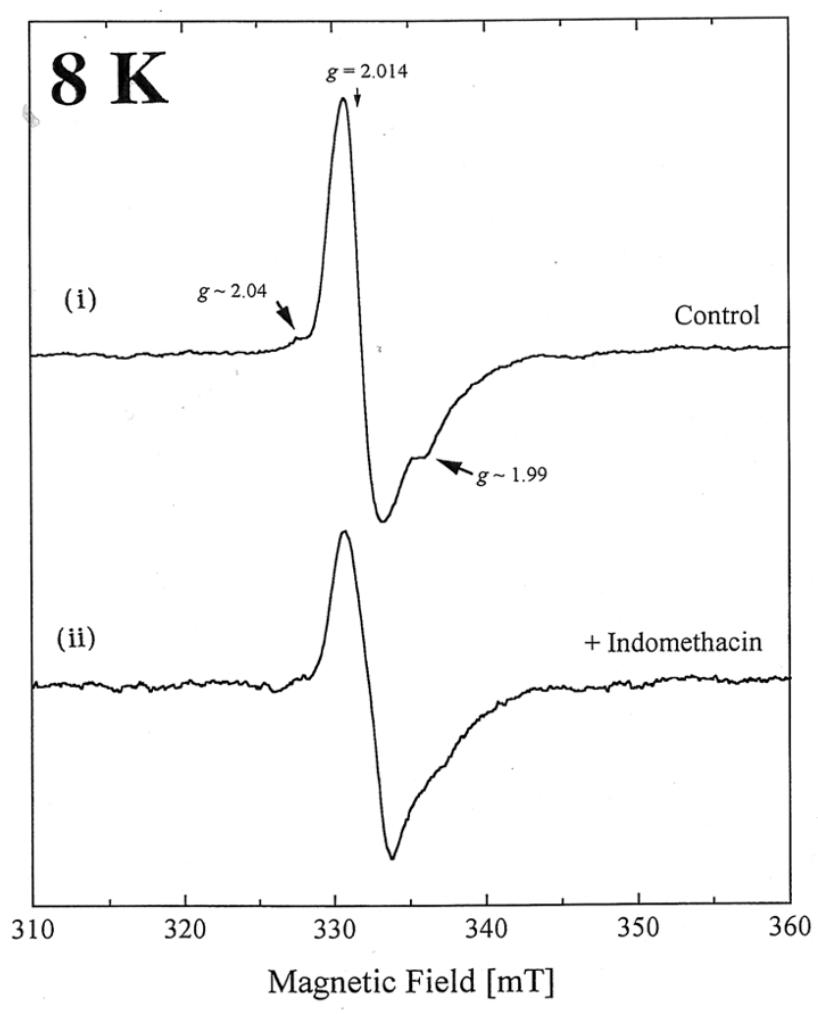

Figure I

The EPR spectra of (I) DMSO-control and (ii) indomethacin (25 $\mathrm{mM}$ final concentration) treated bovine heart submitochondrial particles at $8 \mathrm{~K}$.

\section{Discussion}

The present results show that indomethacin treated rats and not those pretreated with DAHP exhibited small intestinal ulcers at $20 \mathrm{hr}$. There was increased intestinal permeability and an early ( $1 \mathrm{hr}$ ) direct effect of indomethacin consistent with inhibition of electron transport in both groups but only the indomethacin treated group exhbited a haem-nitrosyl complex EPR signal at $g \sim 2.07$. Thus the present result suggests that the nitric oxide radical may play a role in the development of indomethacin-induced ulcers. Within the current pathogenic framework [5] where it is proposed that NSAIDs affect mitochondrial energy metabolism leading to increased intestinal permeability and where the micro vascular alterations, caused by changes in prostaglandin and nitric oxide metabolism are the driving force in the development of ulcers. The involvement of nitric oxide occurs temporarily later or at a different pathophysiological step than the intestinal permeability changes. The non-acidic Pro-NSAID nabumetone caused no intestinal lesion as previously described [22] and was not associated with changes in the EPR spectra, despite being effective inhibitors of cyclooxygenase and associated with decreased mucosal prostaglandins at much smaller doses[22]. These findings are consistent 


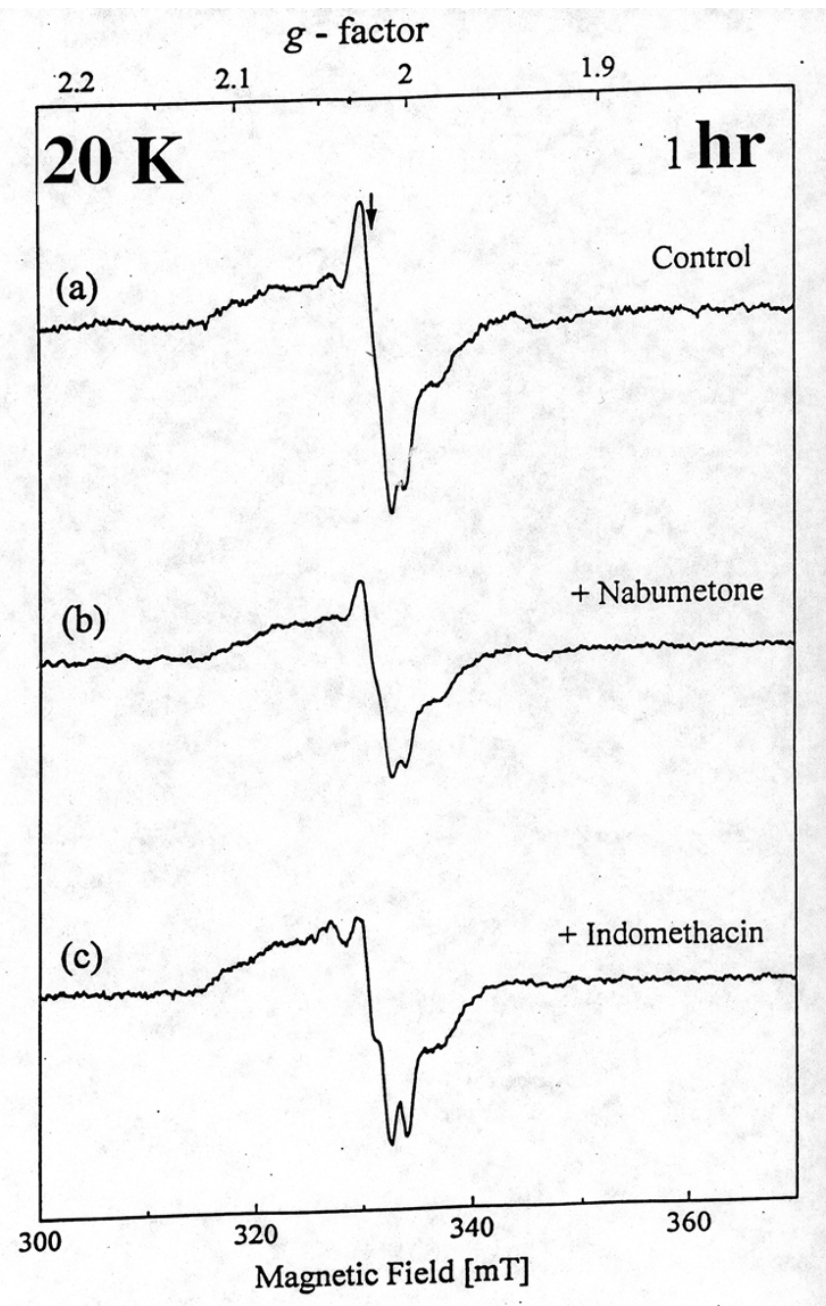

Figure 2

The EPR spectra of rat jejunal homogenates at $20 \mathrm{~K}$ after I $\mathrm{hr}$ oral gavage of (a) DMSO, (b) nabumetone $(500 \mathrm{mg} / \mathrm{kg}$ b.wt) and (c) indomethacin $(20 \mathrm{mg} / \mathrm{kg} \mathrm{b.wt})$

with previous in vitro and in vivo work showing that nabumetone itself does not alter mitochondrial energy metabolism whilst its active metabolite 6MNA does. However it would appear that as 6MNA is formed following absorption of nabumetone, over $99 \%$ of $6 \mathrm{MNA}$ is protein bound within the circulation and it is not excreted in bile, insufficient intestinal concentrations of 6MNA are achieved to affect enterocyte mitochondria.

NSAIDs uncouple oxidative phosphorylation at 30-500 $\mathrm{uM}$ and inhibit mitochondrial electron transport at higher concentration in vitro [8] The relative importance and contribution of uncoupling and inhibition in the pathogenesis of the "topical" Phase of damage is uncertain, but either mechanism would lead to decreased cellular ATP and hence increased intestinal permeability. In this study we assessed the characteristics of NSAID modulation of

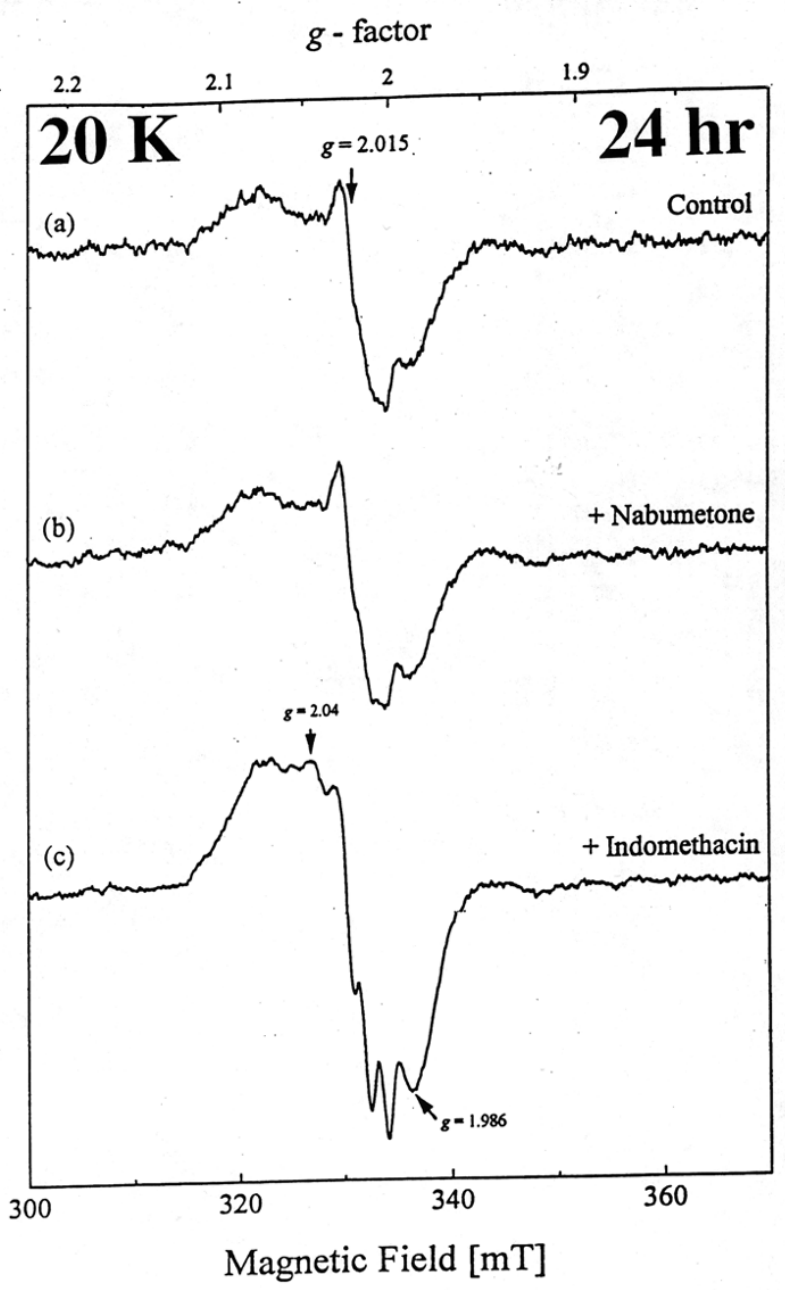

Figure 3

The EPR spectra of rat jejunal homogenates at $20 \mathrm{~K}$ after 24 $\mathrm{hr}$ oral gavage of (a) DMSO, (b) nabumetone $(500 \mathrm{mg} / \mathrm{kg}$ b.wt) and (c) indomethacin (20 mg/kg b.wt)

electron transport in vivo in rats and in vitro in bovine heart submitochondrial particles. The EPR spectra observed at $1 \mathrm{hr}$ would appear to be the result of a direct effect of indomethacin on electron transport unrelated to NO', since pretreatment with DAHP did not alter the indomethacin spectra. The EPR spectra at $24 \mathrm{hr}$ post indomethacin [Fig. 2B (c)] indicated formation of NO' which is further substantiated by abolition of this signal when rats were pretreated with DAHP [Fig. 3].

The activity of iNOS is controlled mainly at the levels of transcription and translation and is dependent on the availability of co-factors including tetrahydrobiopterin (BH4). A recent study has demonstrated that inhibition of BH4 synthesis reduces $\mathrm{NO} \bullet$ production in vivo in experimental endotoxic shock[12]. The same strategy of inhibition of BH4 synthesis has been applied in this present 


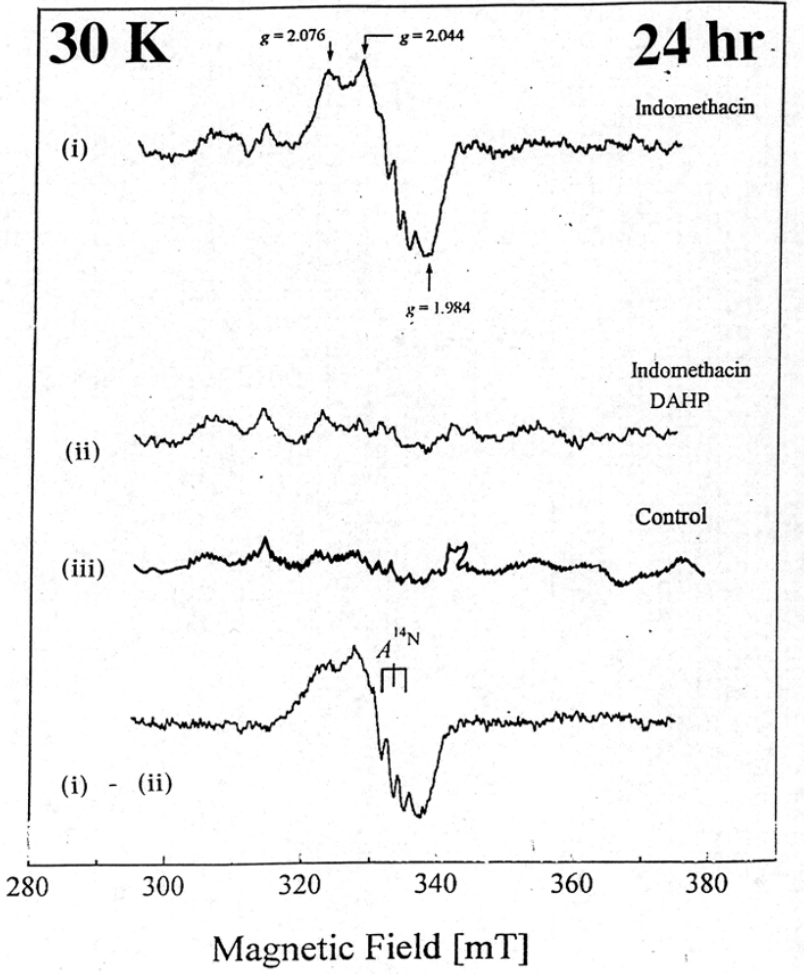

Figure 4

Comparison of EPR spectra of indomethacin and DAHP pretreated indomethacin group at $30 \mathrm{~K}$ after $24 \mathrm{hr}$ treatment.

attempt to investigate ulcer formation in indomethacininduced intestinal ulcer. We found that injection of DAHP an inhibitor of $\mathrm{BH} 4$ synthesis prevented ulcer formation, although it did not alter the increased intestinal permeability induced by indomethacin. This suggests that it does not modulate the direct biochemical actions of NSAIDs but interferes at a later stage in the pathogenesis of the damage. One possibility is that DAHP suppresses NO formation in neutrophils (iNOS), which infiltrate the mucosa in response to increased intestinal permeability. In order to study the specificity of the nitric oxide synthesis the experiments with $\mathrm{N}(\mathrm{G})$-nitro-L-argenine methyl ester (L-NAME-10 mg / kg b wt s.c $1 \mathrm{hr}$ prior to indomethacin) was carried out to prevent nitrosyl signals and ulcers induced by indomethacin. We did not find the inhibition of intestinal ulcers.

This observation is further confirmed by the recent reports [25] stating the worsening effect of L-NAME $(5-20 \mathrm{mg} / \mathrm{kg})$ on these lesions was dose-dependently observed in association with further enhancement of the bacterial translocation and intestinal hypermotility following indomethacin. Thus it has been concluded that this effect may be due to the nonspecific inhibition of L-NAME and

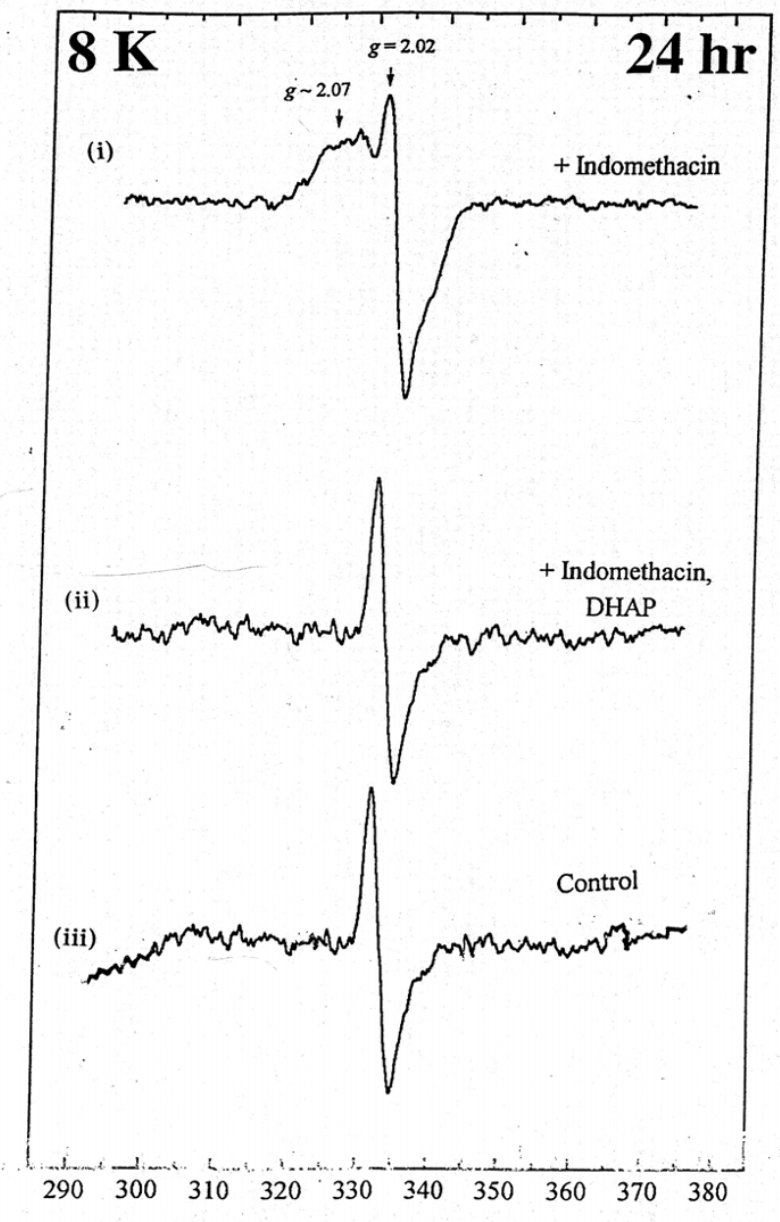

Figure 5

Comparison of EPR spectra of indomethacin and DAHP pretreated indomethacin group at $8 \mathrm{~K}$ after $24 \mathrm{hr}$ treatment.

it is not a specific inhibitor of iNOS cofactor biopterin complex and hence excluded from EPR study.

\section{Conclusions}

In summary, it is interesting that in spite of the increased intestinal permeability, there were no ulcers seen in the DAHP treated group indicating that inhibition of inducible nitric oxide synthase (in neutrophils and macrophages) could become a targeted therapeutic approach to reduce the intestinal toxicity of NSAIDs, and nabumetone is comparatively safer with neither inducing ulcer nor formation of nitrosyl complex in this study.

\section{Competing interests}

None declared 


\section{Authors' contributions}

SS carried out the planning, animal experimentations, EPR measurements and drafted the manuscript. RS contributed for drafting the manuscript. SR carried out submitochondrial particle isolation and In vitro NSAIDs inhibition study. JKS helped for the computation of EPR spectra and contributed in the interpretation of the spectra. JW and IB conceived of the study and participated in its design, coordination, corrections and helping to draft this manuscript.

\section{Grant Support}

This work was supported by an unconditional grant from Smith Kline Beecham, USA to Dr. S. Somasundaram.

\section{Abbreviations}

EPR, electron paramagnetic resonance; NSAIDs, nonsteroidal anti-inflammatory drugs; DAHP, 2,4-diamino-6-hydroxy pyrimidine; DMSO, dimethyl sulfoxide

\section{Acknowledgements}

The authors thank Professor. R. Cammack for providing electron paramagnetic resonance spectroscope for the above study.

\section{References}

I. Barrier $\mathrm{CH}$, Hirschowitz $\mathrm{BI}$ : Current controversies in rheumatology. Controversies and management of nonsteroidal antiinflammatory drug induced side effects on upper gastrointestinal tract. Arthritis Rheum 1989, 32:926-929

2. Bjarnason I, Hayllar J, Macpherson AJ, Russel AS: Side effects of nonsteroidal anti-inflammatory drugs on the small and large intestine. Gastroenterology 1993, 104:1832-1847

3. Whittle BJR: Mechanism underlying gastric mucosal damage induced by indomethacin and bile salt, and the actions of prostaglandins. Br J Pharmacol 1977, 60:455-460

4. Whittle BJR: Protective mechanisms of gastric mucosa. In The Stomach 1992, 81-101

5. Somasundaram S, Hayllar J, Rafi S, Wrigglesworth J, Macpherson A, Bjarnason I: The biochemical basis of NSAID-induced damage to the gastrointestinal tract: A review and a hypothesis. Scandinavian J Gastroenterology, 1995, 30:289-299

6. Whitehouse MW: Biochemical properties of anti-inflammatory drugs-III. Uncoupling of oxidative phosphorylation in a connective tissue (cartilage) and liver mitochondria by salicylate analogues: relationship of structure to activity. Biochem Pharmacol 1964, 13:319-336

7. Somasundaram S, Hayllar J, Bjarnason I: Effect of indomethacin on rat jejunal ATP, antioxidants as well as structure and function of T84 cell line. Gastroenterology 2000, I I 8:A248

8. Somasundaram S, Sigthorsson G, Simpson RJ, Watts J, Jacob M, Tavares IA, Rafi S, Roseth A, Foster R, Price AB, Wrigglesworth JM, Bjarnason I: Uncoupling of intestinal mitochondrial oxidative phosphorylation and inhibition of cyclooxygenase are required for the development of NSAID-enteropathy in the rat. Aliment Pharmacol Ther. 2000, 14:639-50

9. Somasundaram S, Rafi S, Hayllar J, Sigthorsson G, Jacob M, Price $A B$, Macpherson A, Mahmod T, Scott D, Wrigglesworth JM, Bjarnason I: Mitochondrial damage: a possible mechanism of the "topical" phase of NSAID induced injury to the rat intestine. Gut 1997, 4 I:344-53

10. Salzman AL: Nitric Oxide in the Gut. New Horizons 1995, 3:33-45

II. Evans SM, Laszlo F, Whittle BJR: Nonsteroidal anti-inflammatory drugs induce nitric oxide synthase and microvascular injury in rat jejunum. A role of peroxynitrite. Gastroenterology 1996, I 10:A 104

12. Bune AJ, Brand MP, Heales SJR, Shergill JK, Cammack R, Cook HT: Inhibition of tetrahydrobiopterin synthesis reduces invivo
Nitric oxide production in experimental endotoxic shock. $\mathrm{Bi}$ ochem. Biophys. Res. Comm. 1996, 220:13-19

13. Tepperman BL, Brown JF, Whittle BJ: Nitric oxide synthase induction and intestinal epithelial cell viability in rats. Am. J Physiology 1993, 265:G214-G218

14. Tepperman BL, Brown JF, Korolkiewicz R, et al: Endotoxin challengepromotes neutrophill-independentnitric oxide synthase induction, injury, and CGMP formation in rat colonic epithelial cells. Abstr. Gastroenterology 1994, I 06:A782

15. Hall DM, Buettner GR, Matthes RD, Gisolfi CV: Hyperthermia stimulates nitric oxide formation: electron paramagnetic resonance detection of NO'-heme in blood. J. Appl. Physiol. 1994, 77:548-553

16. Cleeter MWJ, Cooper JM, Darley-Usmar VM, Moncada S, Schapira AHV: Reversible inhibition of cytochrome c oxidase, the terminal enzyme of mitochondrial respiratory chain, by nitric oxide. Implications of neurodegenerative diseases. FEBS letters. 1994, 345:50-54

17. Hayllar J, Somasundaram S, Saratchandra P, Levi AJ, Bjarnason I: Early cellular events in the pathogenesis of NSAID enteropathy in the rat. Gastroenterology I99I, I 00:A586

18. Anthony A, Dhillon AP, Nygard G, Hudson M, Piasecki C, Strong P, Trevethik MA, Clayton NM, Jordan CC, Pounder RE, Wakefield AJ: Early histological features of small intestinal injury induced by indomethacin. Aliment Pharmacol. Ther 1993, 7:29-40

19. Melarange R, Gentry C, Connel O, Blower PR, Neil C, Kelwin AS, Toseland CD: Anti-inflammatory and gastrointestinal effects of nabumetone or its metabolite, 6-methoxy-2-naphthylacetic acid (6MNA). Comparative study with indomethacin. Dig. Dis. Sci. 1992, 37:1847-1852

20. Schneider WC, Hogeboom GH: Intracellular distribution of enzymes V. Further studies on the distribution of cytochrome c in rat liver homogenates. J. Biol. Chem. 1950, I 83:123-I 28

21. Yonetani T, Yamamoto H, Erman JE, Leigh JS Jr, Reed GH: Electromagnetic properties of hemoproteins $\mathbf{V}$. Optical and electron paramagnetic resonance characteristics of nitric oxide derivatives of metalloporphyrin-apohemoprotein complexes. J. Biol Chem 1972, 247:2447-55

22. Davies NM, Wright MR, Jamali F: Anti-inflammatory Drug induced small intestinal permeability: The rat is a suitable model. pharmaceutical research. 1994, I I:1652-1656

23. Ruzicka FJ, Beinert H, Schepler KL, Dunham WR, Sands RH: Interaction of ubisemiquinone with a paramagnetic component in heart tissue. Proc. Natl. Acad. Sci. 1975, 72:2886-90

24. Somasundaram S, Rafi S, Jacob M, Sigthorsson G, Mahmud T, Sherwood R, Price AB, Macpherson A, Scott D, Wrigglesworth JM, Bjarnason I: Intestinal tolerability of nitroxybutyl-flurbiprofen in rats. Gut 1997, 40:608-13

25. Tanaka A, Mizoguchi H, Kunikata T, Miyazawa T, Takeuchi K: Protection by constitutively formed nitric oxide of intestinal damage induced by indomethacin in rats. J Physiol. Paris. 200 I, 95:35-41

\section{Pre-publication history}

The pre-publication history for this paper can be accessed here:

http://www.biomedcentral.com/1471-230X/2/8/prepub 